\title{
A KINETIC STUDY OF BULK THERMAL POLYMERIZATION OF STYRENE
}

\author{
KUNIO ARAI, HiRomi YAMAGUCHI, SHozABURo SAITO \\ Department of Chemical Engineering, Tohoku University, Sendai 980 \\ EIJI SARASHINA AND TAKASHI YAMAMOTO \\ Central Research Laboratory, Idemitsu Kosan Co., Ltd., Sodegaura 292-01
}

Key Words: Thermal Polymerization, Styrene, Polymerization Rate, Gel Effect, Simulation Model

\begin{abstract}
A kinetic study was performed on the bulk thermal polymerization of styrene. The conversion and average molecular weights were measured. Rate equations were derived on the basis of a model proposed in a previous paper which introduced the gel effect into each elementary reaction by considering the decrease of segmental jump frequency during polymerization. The model could successfully simulate the conversion and the average molecular weight.
\end{abstract}

\section{Introduction}

General-purpose polystyrene is usually produced by bulk thermal polymerization in the temperature range from 100 to $200^{\circ} \mathrm{C}$. Hui and Hamielec ${ }^{3)}$ proposed an empirical equation of the reaction rate for the bulk thermal polymerization of styrene in this temperature range. Their kinetic model gave a satisfactory fit of conversion and molecular weights.

In a previous work, ${ }^{1)}$ we proposed a kinetic model of polymerization by considering the effects of a decrease of a jump frequency of a polymer segment on the rate of each elementary reaction and showed that the model could successfully simulate the conversion and the average molecular weights over the complete course of chemically-initiated bulk polymerization for styrene and methyl methacrylate.

In this work, we attempt to apply this model to the bulk thermal polymerization of styrene. The conversion and average molecular weights were measured under temperatures from 100 to $180^{\circ} \mathrm{C}$.

\section{Experimental}

After monomer (styrene) was purified by the standard methods, ${ }^{1}$ its bulk thermal polymerization was carried out in glass ampoules ( $3.6 \mathrm{~mm}$ i.d.) with about $1 \mathrm{gr}$ of monomer. A thermocouple was inserted into the ampoule and no significant temperature rise was observed. The conversion was determined from a precipitation method. Number and weight average molecular weights were measured at room temperature by means of a gel permeation chromatograph (HPLC fabricated by Waters Associates) having one Shodex A 802 and two Shodex A-80M columns

\footnotetext{
Received March 1, 1986. Correspondence concerning this article should be addressed to $\mathrm{S}$. Saito.
}

arranged in a series with UV detector. Tetrahydrofuran (THF) was used as an elution solvent at a flow rate of $1 \mathrm{~cm}^{3} / \mathrm{min}$. A $10 \mathrm{mg}$ sample was dissolved in $10 \mathrm{~cm}^{3}$ of the THF and $2 \mathrm{~cm}^{3}$ was injected into the HPLC. For analysis of the elution curves we used a calibration curve made from standard polystyrene samples from Waters Associates.

\section{Model Development}

\subsection{Initiation}

According to Pryor and Lasswel1 ${ }^{5)}$ and Pryor and Coco, ${ }^{4)}$ the thermal initiation of styrene is given as follows.

$$
\begin{aligned}
& M+M \stackrel{k_{1}}{\rightleftharpoons} \\
& M+M \stackrel{k_{2}}{\underset{k_{-2}}{\rightleftharpoons}} \mathrm{phC} \mathrm{HCH}_{2} \mathrm{CH}_{2} \mathrm{C} \mathrm{Hph} \\
& \text { phCHCH}{ }_{2} \mathrm{CH}_{2} \dot{\mathrm{CH}} \mathrm{Hph} \stackrel{k_{\mathrm{cyc}}}{\longrightarrow} 1,2- \\
& \text { diphenylcyclobutane }
\end{aligned}
$$

$$
\begin{aligned}
& M+\mathrm{AH} \stackrel{k_{i}}{\longrightarrow} \mathrm{phCHCH}_{3}(\dot{\mathrm{M}})+\mathrm{H}_{\mathrm{ph}} \\
& M+\mathrm{AH} \stackrel{k_{\mathfrak{c}}}{\longrightarrow} \text { trimer } \\
& \dot{A} \underset{\text { very fast }}{\stackrel{\text { aromatization }}{\longrightarrow}}(\dot{A}) \\
& \dot{R}_{j}+\mathrm{AH} \stackrel{k_{f \mathrm{AH}}}{\longrightarrow} P_{j}+\dot{R}_{1} \\
& \dot{A}+M \stackrel{k_{\mathrm{A}}}{\longrightarrow} \dot{R}_{1} \\
& \dot{M}+M \stackrel{k_{\mathrm{B}}}{\longrightarrow} \dot{R}_{1}
\end{aligned}
$$


Williams ${ }^{8)}$ pointed out that the reaction of Eq. (4) is subjected to the following cage effect.

$$
\begin{aligned}
& \mathrm{AH}+M \stackrel{k_{i}}{\longrightarrow}[\dot{A}+\dot{M}] \\
& {[\dot{A}+\dot{M}] } \stackrel{\text { diffusion }}{\longrightarrow} \dot{A}+\dot{M} \\
& \stackrel{\text { combination }}{\longrightarrow} A \text {-sty } \\
& \stackrel{\text { disproportionation }}{\longrightarrow} \mathrm{phT}+M
\end{aligned}
$$

Since Eq. (4) is considered to be the rate determining step for the initiation process, the rate of initiation is given by

$$
r_{i}=2 f k_{i}[\mathrm{AH}][\mathrm{M}]
$$

where $f$ expresses thermal initiation efficiency (abbreviated as initiator efficiency). Applying the stationary state hypothesis to AH, Eq. (14) is transformed into the following equation.

$$
r_{i}=\frac{2 f k_{i} k_{1}[\mathrm{M}]^{3}}{k_{-1}+\left(k_{c}+k_{i}\right)[\mathrm{M}]+k_{f A \mathrm{H}}\left(r_{i} / k_{t}\right)^{1 / 2}}
$$

Assuming the term $k_{f \mathrm{AH}}\left(r_{i} / k_{t}\right)^{1 / 2}$ to be negligible, the rate of initiation is obtained as follows:

$$
r_{i}=f_{\frac{k_{3}}{\bar{k}_{2}}+\bar{k}_{2}[\mathrm{M}]}
$$

where

$$
\begin{aligned}
& \bar{k}_{2}=\frac{k_{i}+k_{c}}{2 k_{i} k_{1}} \\
& \bar{k}_{3}=\frac{k_{-1}}{2 k_{i} k_{1}}
\end{aligned}
$$

The values of $\overline{k_{2}}$ and $\overline{k_{3}}$ are determined by fitting Eq. (16) to the initial rate of polymerization, $r_{p 0}$, and the following equation is obtained.

$$
r_{i}=f \frac{1.548 \times 10^{9}[\mathrm{M}]^{3}}{1+0.625[\mathrm{M}]} \exp (-14050 / T)
$$

As shown in Fig. 1, good correlation is obtained. As in the previous paper, ${ }^{1)}$ initiator efficiency is estimated from the following equations:

$$
\begin{aligned}
& f=0.47 f_{p} /\left(1-0.53 f_{p}\right) \\
& f_{p}=2 \phi_{\mathrm{I}} /\left(2 \phi_{\mathrm{I}}+\phi_{\mathrm{I}, \text { react }}\right)
\end{aligned}
$$

For convenience of calculation, Eq. (21) is converted into the following form:

$$
f_{p}=\frac{2 \phi_{\mathrm{I}} / \phi_{\mathrm{I} 0}}{2 \phi_{\mathrm{I}} / \phi_{\mathrm{I} 0}+\phi_{\mathrm{I}, \mathrm{reac}} / \phi_{\mathrm{I} 0}}
$$

Jump frequency of initiator radicals, $\phi_{1}$, was estimated from Bueche's free volume theory ${ }^{2)}$ in the previous paper. However, recently Vrentas et al. ${ }^{6,7)}$

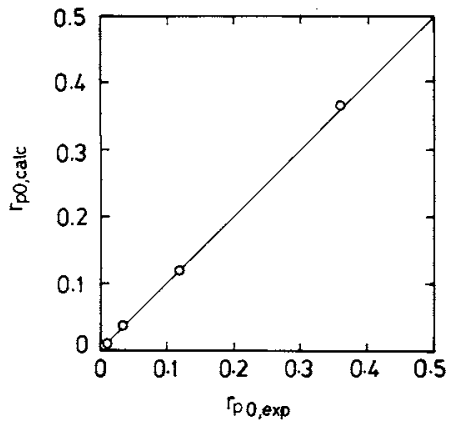

Fig. 1. Comparison between calculated and experimental initial rate of polymerization.

proposed a modification of Bueche's theory of diffusion. According to this theory the concentration dependence of the self-diffusion coefficient of monomer in polymer solution, $D_{1}$, can be described as follows:

$$
D_{1}=D_{01} \exp \left\{\frac{-\gamma\left(w_{1} \hat{V}_{1}^{*}+w_{2} \xi \hat{V}_{2}^{*}\right)}{\hat{V}_{f}}\right\}
$$

the quantity $\xi$ is defined by the ratio of the critical volume per mole of solvent, $\hat{V}_{1}^{*} M_{1}$, to that of the jumping units of a polymer, $\hat{V}_{2}^{*} M_{j}$, where $M_{1}$ is the molecular weight of the solvent and $M_{j}$ is that of jumping units. $\gamma$ is an overlap parameter whose value is probably between 0.5 and 1. By using Eq. (23), $\phi_{\mathrm{I}} / \phi_{\mathrm{IO}}$ can be expressed as

$$
\phi_{\mathrm{I}} / \phi_{\mathrm{I} 0}=\exp \left\{\frac{\gamma \hat{\mathrm{V}}_{1}^{*}}{\hat{\mathrm{V}}_{f 0}}-\frac{\gamma\left(w_{1} \hat{V}_{1}^{*}+w_{2} \xi \hat{V}_{2}^{*}\right)}{\hat{V}_{f}}\right\}
$$

By modifying the results for the ethylbenzenepolystyrene system presented by Vrentas et al., the values of $\hat{V}_{1}^{*}, \hat{V}_{2}^{*}$ and $\xi$ are redetermined as follows:

$$
\hat{V}_{1}^{*}=0.9\left[\mathrm{~cm}^{3} / \mathrm{g}\right], \hat{V}_{2}^{*}=0.85\left[\mathrm{~cm}^{3} / \mathrm{g}\right], \xi=0.7
$$

while the overlap parameter, $\gamma$, is treated as an adjustable parameter. $\phi_{\mathrm{I}, \mathrm{reac}} / \phi_{\mathrm{I} 0}$ can be expressed as

$$
\phi_{1, \text { react }} / \phi_{\mathrm{IO}}=C \exp \left(-\frac{E_{\text {react }}-E_{D}}{R T}\right)
$$

where $R$ is the gas constant and the activation energies of recombination, $E_{\text {react, }}$ and diffusion, $E_{D}$ are assumed to be equal to those of the termination of polymerization of styrene and the self-diffusion of benzene molecule, respectively. That is:

$$
E_{\text {react }}-E_{D}=-1600[\mathrm{cal} / \mathrm{mol}]
$$

The constant $C$ in Eq. (26) is determined by using the initiator efficiency at the initial stage of polymerization at $100^{\circ} \mathrm{C}\left(f_{0}=0.1\right)$ :

$$
C \fallingdotseq 0.98
$$

\subsection{Propagation and chain transfer}

The mechanism of propagation reaction has been described previously ${ }^{11}$ and the chain transfer reaction 
to monomer is introduced into the bulk thermal polymerization of styrene.

\subsection{Termination reaction}

According to the previous paper, ${ }^{1)}$ the overall rate constant of termination, $k_{t}$, is given as

$$
k_{t}=k_{t c} k_{t D} /\left(k_{t c}+k_{t D}\right)
$$

and the diffusion-controlled rate constant, $k_{t D}$, was derived from Bueche's free volume theory by considering entanglements between polymer chains. In this work, the theory presented by Vrentas et al. is adopted. That is,

$$
k_{t D}=A \psi \exp \left\{\frac{-\gamma\left(w_{1} \hat{V}_{1}^{*}+w_{2} \xi \hat{V}_{2}^{*}\right)}{\hat{V}_{f} \xi}\right\}
$$

where $A$ is a model parameter which is on the order of $10^{13} \sim 10^{16}$ and $\psi$ represents the effect of entanglements between polymer chains on $k_{t D}$ and is expressed by

$$
\begin{array}{ll}
\psi=1 & w_{2}<w_{2 e} \\
\psi=\left(b_{0} / \varepsilon_{e}\right) /\left(1+b_{0} / \varepsilon_{e}\right) & w_{2}>w_{2 e}
\end{array}
$$

The value of $w_{2 e}$ is probably nearly equal to zero from the literature. ${ }^{1)}$ It is, however, more appropriate to consider that with increasing temperature, entanglement points are not bounded at fixed points but traverse freely in the system. Then $\varepsilon_{e}$ may be proportional only to the number of entanglement points per unit volume $n_{\text {ent }}$. Since $n_{\text {ent }}$ is considered to be proportional to the mass of polymer entangled with other polymers in unit volume, $v_{e}$, Eq. (32) is rewritten as

$$
\psi=\left(b / v_{e}\right) /\left(1+b / v_{e}\right) \quad \text { at } \quad w_{2}>w_{2 e}
$$

where $b$ is a model parameter and $v_{e}$ is calculated from the following equation.

$$
v_{e}=\left(w_{2}-w_{2 e}\right) \int_{J_{c}}^{\infty} g(j) d j
$$

where $J_{c}$ is critical chain length and can be estimated as follows:

$$
J_{c}=J_{b}\left(w_{2}-w_{2 e}\right) / 2
$$

For $g(j)$ in Eq. (34), which represents the weight fraction distribution of product polymer, the following logarithmico-normal distribution is used.

$$
\begin{gathered}
\int_{J_{c}}^{\infty} g(j) d j=\frac{1}{2}\{1-\operatorname{erf}(u)\} \\
u=\frac{1}{\beta_{0}}\left(\frac{J_{c}}{J_{0}}\right), \quad J_{0}=\sqrt{\bar{J}_{N} \bar{J}_{w}}, \quad \beta_{0}=2\left(\ln \frac{\bar{J}_{w}}{J_{0}}\right)^{1 / 2}
\end{gathered}
$$

\subsection{The kinetic equation}

1) Initial reaction Initial reactions are given by
Eqs. (1) to (13). The propagation, termination, and chain transfer reactions are:

2) Propagation reaction

$$
\begin{gathered}
R_{j}^{*}+M_{E} \stackrel{k_{p c}\left(1-P_{E}(0)\right)}{\longrightarrow} R_{j+1}^{*} \\
\stackrel{k_{p c} P_{E}(0)}{\longrightarrow} \mathscr{R}_{j+1} \\
\mathscr{R}_{j+1} \stackrel{k_{\text {Diff }}^{\longrightarrow}}{\longrightarrow} R_{j+1}^{*}
\end{gathered}
$$

3) Termination reaction

$$
\begin{aligned}
& R_{i}+R_{j} \stackrel{k_{i}}{\longrightarrow} P_{i+j} \\
& R_{i}^{*}+R_{j}^{*} \stackrel{k_{i p}}{\longrightarrow} P_{i+j}
\end{aligned}
$$

4) Chain transfer reaction to monomer

$$
\begin{gathered}
R_{i}^{*}+M_{E} \stackrel{k_{f m c}\left(1-P_{E}(0)\right)}{\longrightarrow} P_{j}+R_{1}^{*} \\
\stackrel{k_{f m c} P_{E}(0)}{\longrightarrow} P_{j}+\mathscr{R}_{1}
\end{gathered}
$$

Here, $k_{\text {Diff }}$ is expressed by using the Vrentas theory.

$$
k_{\text {Diff }}=\phi_{0} \exp \left\{-a \gamma\left(w_{1} \hat{V}_{1}^{*}+w_{2} \xi \hat{V}_{2}^{*}\right) / V_{f} \xi\right\}
$$

$\phi_{0}$ is frequency factor of jumps of polymer segment and can be related to a model parameter $A$ by

$$
\phi_{0}=\frac{A \times N_{A} \times 10^{3}}{8 V_{M} \times 60}
$$

The parameter $a$ in Eq. (45) is an adjustable parameter introduced to correct the deviation of $k_{\text {Diff }}$ from the average jump frequency of segments because it is considered that the chain terminal segment is more flexible than the other segments and that the end monomer unit of this terminal segment is completely surrounded by polymer chain units. The value of $a$ is probably nearly equal to $1 .^{1)}$

Equation (42) gives the termination process due to the motion of active centers resulting from propagation. This reaction is not significant at high temperature. Details of each elementary reaction are given elsewhere. ${ }^{1)}$

By introducing the $k$-th moments which are defined for the concentrations of polymer radicals and inactive polymers,

$$
\mu_{R}^{k}=\sum_{j=1}^{\infty} j^{k} R_{j}, \quad \mu_{R^{*}}^{k}=\sum_{j=1}^{\infty} j^{k} R_{j}^{*}, \quad \mu_{P}^{k}=\sum_{j=1}^{\infty} j^{k} P_{j}
$$

and we find the following differential equations:

$$
\begin{gathered}
\frac{1}{V} \frac{d\left(V \mu_{R}^{0}\right)}{d t}=r_{i}-k_{t}\left(\mu_{R}^{0}\right)^{2}-k_{t p}\left(\mu_{R^{*}}^{0}\right)^{2} \\
\frac{1}{V} \frac{d\left(V \mu_{R}^{k}\right)}{d t}=r_{i}+k_{p c} M_{E} \sum_{i=1}^{k}\left(\begin{array}{c}
k \\
i
\end{array}\right) \mu_{R^{*}}^{k-i}-k_{t} \mu_{R}^{k} \mu_{R}^{0} \\
-k_{t p} \mu_{R^{*}}^{k} \mu_{\mathrm{R}^{*}}^{0}+k_{f m c} \mu_{R^{*}}^{0} M_{E}-k_{f m c} \mu_{R^{*}}^{k} M_{E}
\end{gathered}
$$




$$
\begin{aligned}
\frac{1}{V} \frac{d\left(\nabla \mu_{R^{*}}^{0}\right)}{d t} & =r_{i}\left(1-P_{E}(0)\right)-k_{p c} P_{E}(0) M_{E} \mu_{R^{*}}^{0} \\
& -k_{t} \mu_{R^{*}}^{0} \mu_{\mathrm{R}}^{0}-k_{t p}\left(\mu_{R^{*}}^{0}\right)^{2} \\
& +k_{\mathrm{Diff}}\left(\mu_{R}^{0}-\mu_{\mathrm{R}^{*}}^{0}\right)-k_{f m c} P_{E}(0) M_{E} \mu_{\mathrm{R}^{*}}^{0} \\
\frac{1}{V} \frac{d\left(V \mu_{R^{*}}^{k}\right)}{d t}= & r_{i}\left(1-P_{E}(0)\right)-k_{p c} P_{E}(0) M_{E} \mu_{R^{*}}^{k} \\
& +k_{p c}\left(1-P_{E}(0)\right) M_{E} \sum_{j=1}^{\infty}\left(\begin{array}{c}
k \\
i
\end{array}\right) \mu_{R^{*}}^{k-i} \\
& -k_{t} \mu_{R^{*}}^{k} \mu_{R}^{0}-k_{i p} \mu_{R^{*}}^{k} \mu_{R^{*}}^{0}+k_{\mathrm{Diff}}\left(\mu_{R}^{k}-\mu_{R^{*}}^{k}\right) \\
& -k_{f m c} M_{E} \mu_{R^{*}}^{k}+k_{f m c}\left(1-P_{E}(0)\right) M_{E} \mu_{R^{*}}^{0} \\
\frac{1}{V} \frac{d\left(V m_{0}\right)}{d t}= & r_{i}-\frac{1}{2}\left\{k_{t}\left(\mu_{R}^{0}\right)^{2}+k_{t p}\left(\mu_{R^{*}}^{0}\right)^{2}\right\}+k_{f m c} \mu_{R^{*}}^{0} M_{E} \\
\frac{1}{V} \frac{d\left(V m_{1}\right)}{d t}= & r_{i}+k_{p c} M_{E} \mu_{R^{*}}^{0}+k_{f m c} M_{E} \mu_{R^{*}}^{0} \\
\frac{1}{V} \frac{d\left(V m_{k}\right)}{d t} & =r_{i}+k_{p c} M_{E} \sum_{i=1}^{k}\left(\begin{array}{l}
k \\
i
\end{array}\right) \mu_{R^{*}}^{k-i} \\
& +\frac{1}{2} \sum_{i=1}^{k-1}\left(\begin{array}{l}
k \\
i
\end{array}\right)\left(k_{t} \mu_{R}^{k-i} \mu_{R}^{i}+k_{t p} \mu_{R^{*}}^{k-i} \mu_{R^{*}}^{i}\right) \\
& +k_{f m c} M_{E} \mu_{R}^{0}
\end{aligned}
$$

where $m_{k}$ is the $k$-th moment of the product polymer and is equal to the sum of $\mu_{R}$ and $\mu_{P^{\prime}}$, and $V$ is the volume of the reaction system, given by:

$$
V=V_{0}(1-\beta x)
$$

In Eq. (47), $R$ represents the total concentration of polymer radicals and is equal to the sum of $R_{j}^{*}$ and $\mathscr{R}_{j}$.

By definition, the conversion of monomer to polymer, $x$, the number-average degree of polymerization, $\bar{J}_{N}$, and the weight-average degree of polymerization, $\bar{J}_{w}$ are related to $m_{k}$ in the following way.

$$
x=\frac{m_{1} V}{M_{0} V_{0}}, \quad \bar{J}_{N}=\frac{m_{1}}{m_{0}}, \quad \bar{J}_{w}=\frac{m_{2}}{m_{1}}
$$

By adopting the steady-state assumption for radical concentration, the differential equations for $m_{k}$ are solved numerically with the Runge-Kutta method and $x, \bar{J}_{N}$ and $\bar{J}_{w}$ are obtained.

\section{Application of the Simulation Model to Bulk Thermal Polymerization of Styrene}

\subsection{Simulation of conversion-time curves}

Figures 2 to 6 show the conversion-time curves for bulk thermal polymerization of styrene. The calculated results neglecting the gel effect (abbreviated as Ideal in the figures) deviate from the experimental

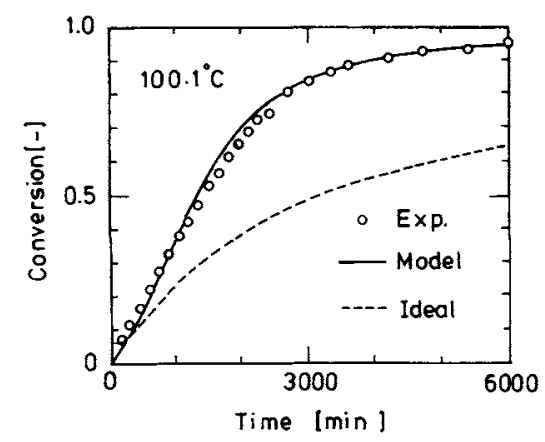

Fig. 2. Conversion vs, time for bulk thermal polymerization of styrene at $100.1^{\circ} \mathrm{C}$.

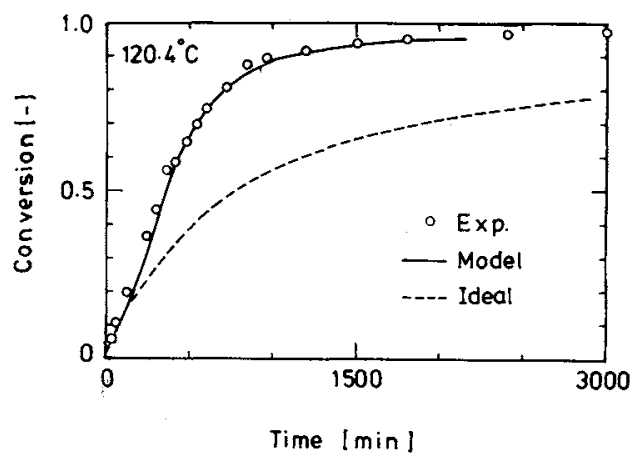

Fig. 3. Conversion vs. time for bulk thermal, polymerization of styrene at $120.4^{\circ} \mathrm{C}$.

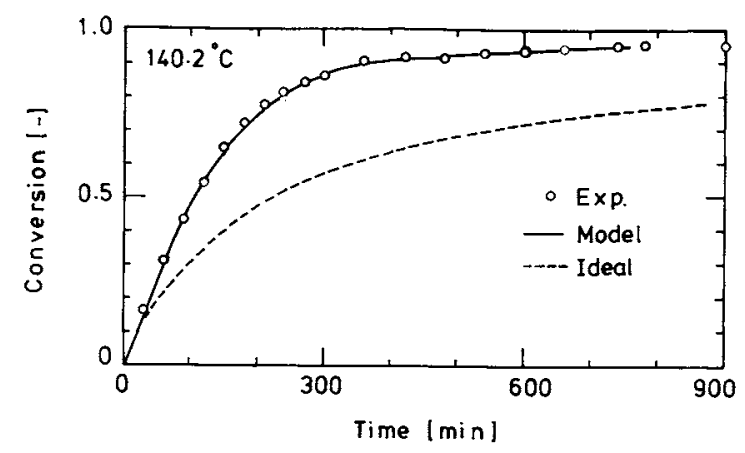

Fig. 4. Conversion vs. time for bulk thermal polymerization of styrene at $140.2^{\circ} \mathrm{C}$.

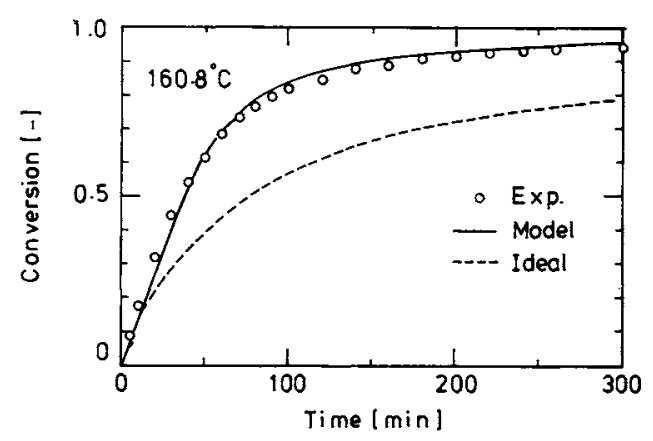

Fig. 5. Conversion vs. time for bulk thermal polymerization of styrene at $160.8^{\circ} \mathrm{C}$.

values as the reaction proceeds. As pointed out by Hui et al., the gel effect, must therefore be considered in the calculation even though reaction temperature is relatively high. Full lines show the calculated results 


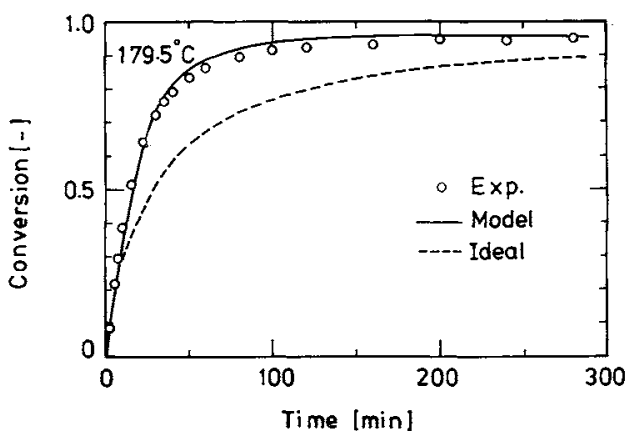

Fig. 6. Conversion vs. time for bulk thermal polymerization of styrene at $179.5^{\circ} \mathrm{C}$.

by the simulation model proposed in this work and good agreement is obtained between the calculated and experimental results. In these calculations, the ajustable parameters were determined at $140^{\circ} \mathrm{C}$. The values of these parameters and those of the rate constants are listed in Tables 1 and 2.

\subsection{Simulation of average molecular weight}

Figures 7 to 11 show comparisons between the measured and calculated average molecular weights. Although some of the measured values of weight average molecular weights are slightly greater than the calculated ones, comparatively good agreement was obtained, taking into account the possible experimental error in analysis of the elution curves. However, for the highest temperature $\left(179.5^{\circ} \mathrm{C}\right)$, both experimental average molecular weights decrease abnormally in the high conversion range. This phenomenon cannot be explained clearly, but it may result from $\beta$-scission of $\mathrm{C}-\mathrm{C}$ bond of the product polymer due to the chain transfer to the carbon atom linked to the phenyl group of polystyrene. In these calculations, chain transfer constant to monomer, $C_{m}$, proposed by Hui et al., is used.

$$
\begin{gathered}
C_{m}=C_{m 0}+B_{1} x \\
B_{1}=-6.91 \times 10^{-4} \log _{10}\left(\frac{473.1008-T}{192}\right)
\end{gathered}
$$

where $C_{m 0}$ represents the chain transfer constant at the initial stage of polymerization and is redetermined from our data of the number-average molecular weight.

$$
C_{m 0}=0.7922 \exp (-2990 / T)
$$

\section{Conclusion}

Data of conversion and average molecular weights in the bulk thermal polymerization of styrene were obtained. By using these data, rate equations were constructed on the basis of a previous model. As a result, conversion and average molecular weights were relatively well correlated. This model theoretically introduces the gel effect into each elementary
Table 1. Adjustable parameters

\begin{tabular}{ccccc}
\hline$a$ & $b$ & $w_{2 e}$ & $A$ & $\gamma$ \\
\hline 0.9 & $1.0 \times 10^{-4}$ & 0.10 & $1.5 \times 10^{4}$ & 0.65 \\
\hline
\end{tabular}

Table 2. Rate constants at initial stage of polymerization and physical properties of polymer and monomer

$$
\begin{array}{rlrl}
k_{p 0}=6.46 \times 10^{8} \exp (-3557 / T) & {[1 / \mathrm{mol} \cdot \mathrm{min}]} \\
\mathcal{k}_{\mathrm{t0}}=7.53 \times 10^{10} \mathrm{exp}(-844 / T) & {[1 / \mathrm{mol} \cdot \mathrm{min}]} \\
\beta= & {[-14]} \\
J_{b}=380.0 & {[-]} \\
\rho_{M}=1.239416 \times 10^{-8} T^{2}-8.780667 \times 10^{-4} \mathrm{~T} \\
& +0.9238228 & {[\mathrm{~g} / \mathrm{ml}]}
\end{array}
$$

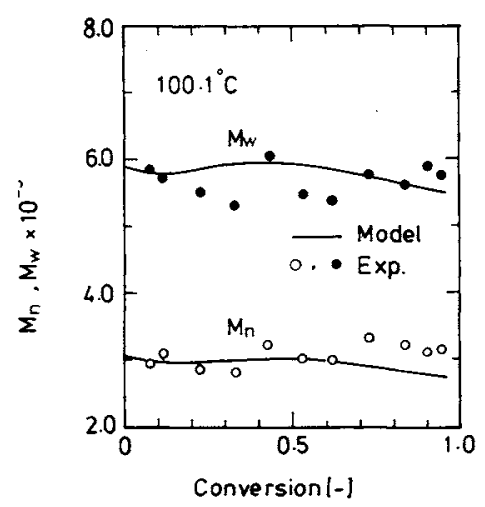

Fig. 7. Average molecular weight vs. conversion for bulk thermal polymerization of styrene at $100.1^{\circ} \mathrm{C}$.

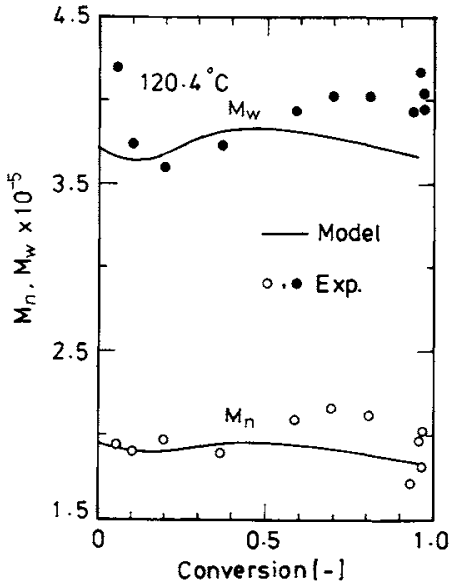

Fig. 8. Average molecular weight vs. conversion for bulk thermal polymerization of styrene at $120.4^{\circ} \mathrm{C}$.

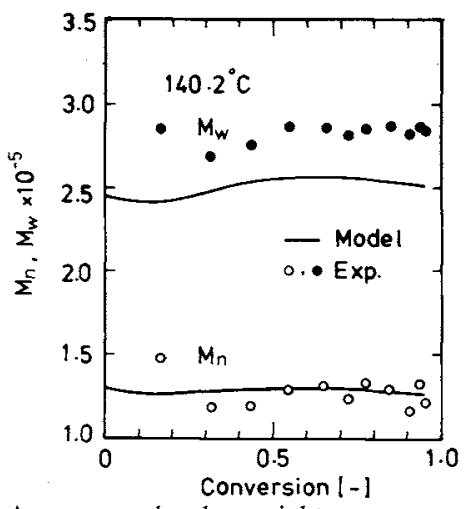

Fig. 9. Average molecular weight vs. conversion for bulk thermal polymerization of styrene at $140.2^{\circ} \mathrm{C}$. 


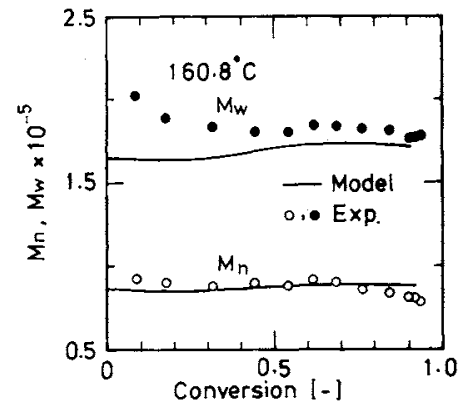

Fig. 10. Average molecular weight vs. conversion for bulk thermal polymerization of styrene at $160.8^{\circ} \mathrm{C}$.

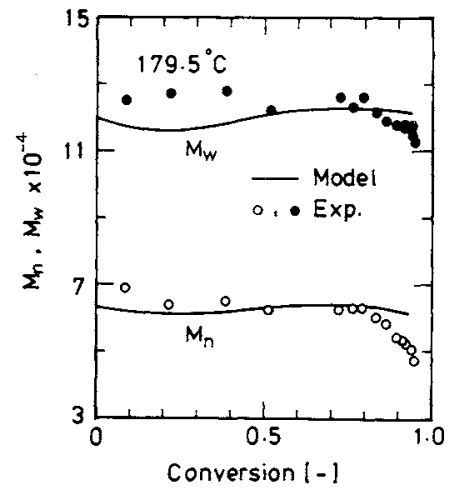

Fig. 11. Average molecular weight vs. conversion for bulk thermal polymerization of styrene at $179.5^{\circ} \mathrm{C}$.

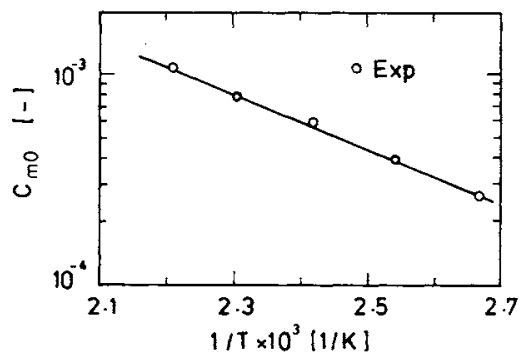

Fig. 12. Temperature dependence of chain transfer constant at initial stage of polymerization.

reaction and may be capable of extension to other polymerization systems such as copolymerization.

\section{Nomenclature}

\begin{tabular}{|c|c|}
\hline$A$ & $=$ model parameter in Eq. (30) \\
\hline$a$ & $=$ model parameter in Eq. (45) \\
\hline$b_{0}$ & $=$ constant in Eq. (32) \\
\hline$b$ & $=$ model parameter in Eq. (33) \\
\hline$B_{1}$ & $=$ model parameter defined by Eq. (58) \\
\hline C & $=$ proportional constant in Eq. (26) \\
\hline$C_{m}$ & $\begin{aligned}= & \text { chain transfer constant of polymer radica } \\
& \text { to monomer }\end{aligned}$ \\
\hline$C_{m 0}$ & $\begin{aligned}= & \text { chain transfer constant at initial stage } \\
& \text { of polymerization }\end{aligned}$ \\
\hline$D_{01}$ & $=$ proportional constant in Eq. (23) \\
\hline$D_{1}$ & $\begin{aligned}= & \text { self-diffusion coefficient of monomer } \\
& \text { in polymer solution }\end{aligned}$ \\
\hline$E_{\text {react }}$ & $=$ activation energy of recombination \\
\hline$E_{D}$ & $=$ activation energy of diffusion \\
\hline & $=$ thermal initiation efficiency \\
\hline
\end{tabular}

$=$ fraction of initiator radicals which escape primary recombination

$=$ weight fraction distribution of product polymer

$=$ critical chain length defined by Eq. (35)

$=$ constant in Eq. (35)

$=$ number average chain length

$=$ weight average chain length

$=$ rate constant in Eq. (1)

$=$ rate constant in Eq. (1)

$=$ rate constant in Eq. (2)

$=$ rate constant in Eq. (2)

$=$ rate constant in Eq. (3)

$=$ rate conştant in Eq. (4)

$=$ rate constant in Eq. (5)

$=$ rate constant in Eq. (7)

$=$ rate constant in Eq. (8)

$=$ rate constant in Eq. (9)

$=$ group of rate constant defined by Eq. (17)

$\bar{k}_{3} \quad=$ group of rate constant defined by Eq. (18)

$=$ chemical reaction-controlled rate constant for termination process

$=$ diffusion-controlled rate constant for termination process

$[1 / \mathrm{mol} \cdot \mathrm{min}]$

$k_{t D}$

$k_{t}$

$k_{t p}$

$=$ rate constant for termination

$[1 / \mathrm{mol} \cdot \mathrm{min}]$

$=$ rate constant for termination due to motion of active centers resulting from propagation

$[1 / \mathrm{mol} \cdot \mathrm{min}]$

- chemical reaction-con

$[1 / \mathrm{mol} \cdot \mathrm{min}]$

$k_{p c}$ for propagation process

$[1 / \mathrm{mol} \cdot \min ]$

$k_{f m c}$

$k_{\text {Diff }}$

$=$ chain transfer constant of polymer radical to monomer

$[1 / \mathrm{mol} \cdot \mathrm{min}]$

$=$ rate constant for $R$ reviving to become $R^{*}$

$M$

$M_{j}$

$=$ monomer and its concentration
$=$ molecular weight of jumping unit

$[1 / \mathrm{min}]$

$=$ molecular weight of solvent

$=$ number average molecular weight

$[\mathrm{mol} / \mathrm{l}]$

$M_{1}$

$M_{n}$
$M_{w}$

$=$ weight average molecular weight

$=$ monomer molecule around a polymer chain end and its concentration

$[\mathrm{g} / \mathrm{mol}]$

$[\mathrm{g} / \mathrm{mol}]$

$M_{E}$

$m_{k}$

$=k$-th moment of size distribution for product polymer

$[\mathrm{g} / \mathrm{mol}]$

$[\mathrm{g} / \mathrm{mol}]$

$=$ initial monomer concentration

$[\mathrm{mol} / \mathrm{l}]$

$M_{0}$

$=$ Avogadro's number

$[\mathrm{mol} / \mathrm{l}]$

$N_{A}$

$P_{E}(0)$

$=$ probability of finding no molecules at the nearest-neighbor cells to a polymer chain end

$[\mathrm{mol} / \mathrm{l}]$

$[-]$

$=$ inactive polymer molecule and its concentration

$=$ initial rate of polymerization $\quad[\mathrm{mol} / \mathrm{l} \cdot \mathrm{min}]$

$=$ polymer radical and its concentration $\quad[\mathrm{mol} / \mathrm{l}]$

$=$ non-growing polymer radical

$=$ growing polymer radical and its concentration

$=$ absolute temperature

$[\mathrm{mol} / \mathrm{l}]$

$=$ time

= free volume per gram of polymer solution

$=$ critical amount of local free volume per gram

$=$ free volume per gram of monomer liquid $[\mathrm{ml} / \mathrm{g}]$

$=$ molar volume of monomer $\quad[\mathrm{ml} / \mathrm{mol}]$

$=$ volume of reaction system at start of polymerization 


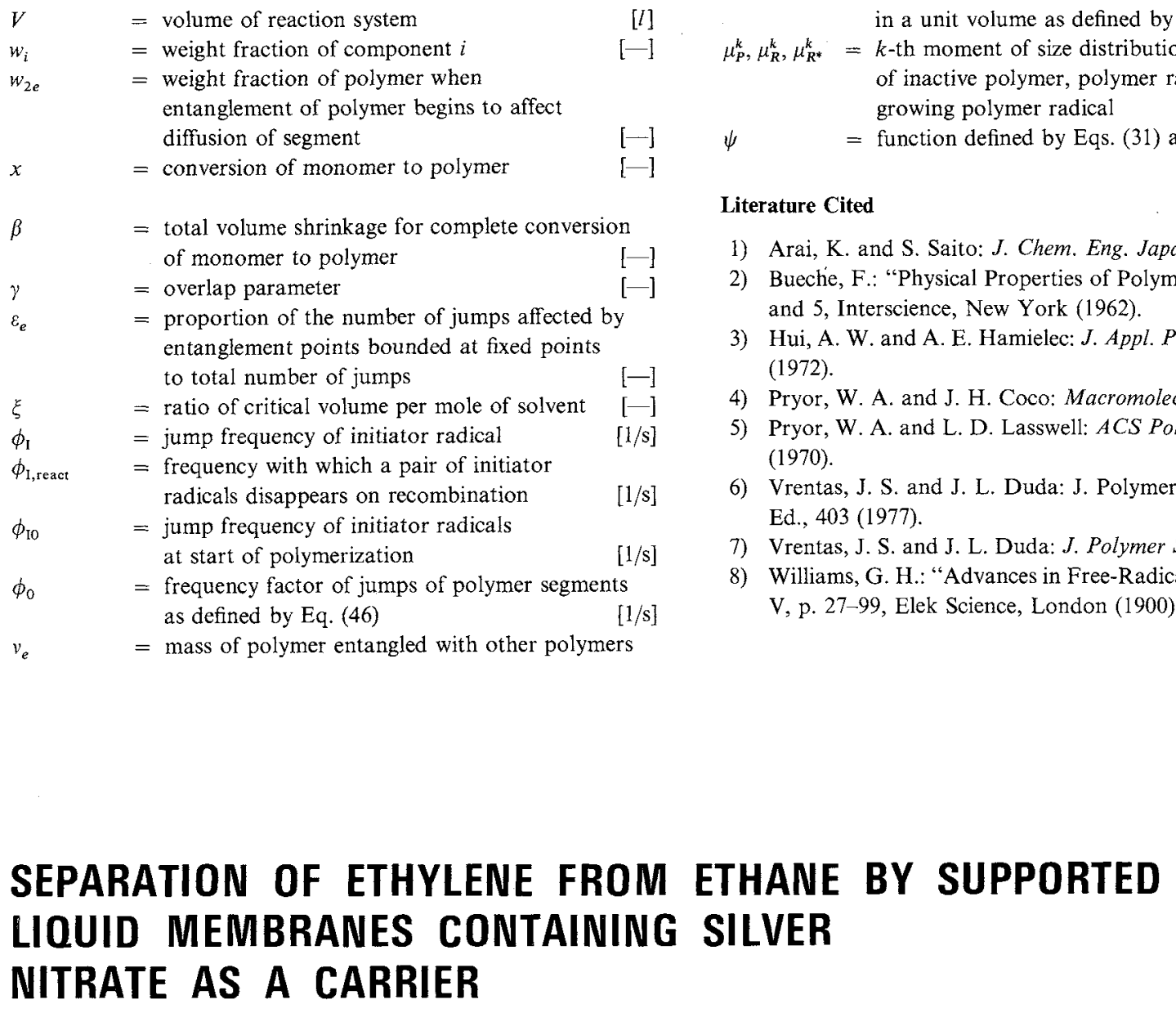

\author{
MASAAKI TERAMOTO, HIDETO MATSUYAMA, \\ TAKUMI YAMASHIRO AND YUJI KATAYAMA \\ Department of Industrial Chemistry, Kyoto Institute of Technology, \\ Kyoto 606
}

\begin{abstract}
Key Words: Facilitated Transport, Liquid Membrane, Ethylene, Silver Nitrate, Carrier Transport, Gas Separation

Experimental results for the facilitated transport of ethylene through supported liquid membranes containing silver nitrate as a carrier are presented. The selectivity factor in the separation of ethylene and ethane increases with increasing carrier concentration, reaching about 1000 when silver nitrate concentration is $4 \mathrm{~mol} / \mathrm{dm}^{3}$. An approximation method for calculating the rate of facilitated transport accompanied by an instantaneous reversible reaction is proposed. This method is applicable even to the case where the diffusivities of the carrier and the complex differ from each other. It is found that the effect of experimental condition on the permeation rate of ethylene is satisfactorily explained by assuming that the reaction between ethylene and $\mathrm{Ag}^{+}$is instantaneous and reversible.
\end{abstract}

\section{Introduction}

Gas separation technique using carrier-mediated or facilitated transport has been attracting increasing attention because very high selectivity can be easily obtained by incorporating a carrier into a membrane

Received April 4, 1986. Correspondence concerning this article should be addressed to M. Teramoto. Y. Katayama is at Asahi Glass Co., Ltd., Ichihara 290. which can selectively and reversibly bind the permeant species to be transported. From this point of view, a number of studies have been presented on the permeation of such gases as carbon monoxide, carbon dioxide, hydrogen sulfide, nitric oxide and oxygen. These works were reviewed and discussed by Kimura et $a .^{2)}$ and Way et al. ${ }^{13)}$

Concerning the separation of olefinic gases from 